\title{
FINITE ELEMENT MODELING OF THE NEURON- ELECTRODE INTERFACE: SEALING RESISTANCE AND STIMULUS TRANSFER AT TRANSITIONS FROM COMPLETE TO DEFECT SEALING
}

\author{
J.R. Buitenweg*, W.L.C.Rutten ${ }^{*}$ and E. Marani** \\ *Institute for Biomedical Technology, Faculty of Electrical Engineering, University of \\ Twente, P.O. Box 217, 7500 AE Enschede, The Netherlands \\ ** Dept. of Physiology, Faculty of Medicine, University of Leiden, P.O.Box 9604, \\ 2300 RC Leiden, The Netherlands \\ "Email: j.r.buitenweg@el.utwente.nl
}

\begin{abstract}
The quality of the electrical contact between a cultured neuron and a substrate embedded microelectrode is of importance for effective transfer of an extracellular applied stimulus current to the intracellular potential. It is affected by the resistance of the seal, i.e. the gap between the cell membrane and the substrate, which restricts the leakage current, thereby favouring the efficiency of the stimulation current.

The effects of variations in the geometry of the neuronelectrode interface on the sealing resistance and on the stimulus transfer are studied using a finite element model of this interface. Variations in the geometry of the neuron-electrode interface are represented by the excentricity, $x_{c}$, of a pillbox shaped neuron with radius $r_{c}$, cultured on an electrode with radius $r_{\mathrm{e}}$.

The results indicate a sharp decrease in both sealing resistance and stimulus transfer when a transition occurs from complete sealing to defect sealing. At that point the leakage current splits up into a current through the gap and a current through the sealing defect.
\end{abstract}

Keywords - Cultured neurons, multi-electrode arrays, Neuron-electrode interface, sealing resistance, stimulus transfer, finite element modeling

\section{INTRODUCTION}

Planar substrate electrode arrays (PEAs) offer the possibility to contact neuronal cultures with a large number of cell-size electrodes [1][2]. In our project, PEAs are used as a tool for the development of a cultured neuron probe, a new type of neuro-electronic interface for highly selective stimulation purposes.

For effective transfer of an extracellular applied stimulus current to the intracellular potential, the quality of the electrical contact between a cultured neuron and an electrode is of importance [3]. This quality is affected by the resistance of the seal, i.e. the gap between the cell membrane and the substrate, which restricts the leakage current, thereby favouring the efficiency of the stimulation current (fig. 1a).

In previous work, a method for measurement of the sealing resistance in the neuron-electrode interface was proposed as a first step towards assessment of the quality of this contact [4]. This method was based on a model of a neuron-electrode interface geometry in which the electrode was completely covered, i.e. sealed by the neuron.

Now, this model is extended to include the effects of varying neuron-electrode geometries on the transfer of extracellular applied stimuli. Variations in the geometry of the neuronelectrode interface are represented as an excentricity, $x_{c}$, of a neuron with radius $r_{c}$, cultured on an electrode with radius $r_{e}$ (fig. 1b). When $x_{c}$ increases from zero, the sealing resistance will decrease due to the altering geometry of the gap between the cell membrane and the substrate. At $x_{c}=r_{c}-r_{e}$, a transition occurs from complete sealing to defect sealing: The leakage current splits up into a current through the gap and a current through the sealing defect. Further increase of $x_{c}$ will also decrease the area of the patch membrane (fig. 1c).

The effects of the neuron excentricity on the sealing resistance and on the stimulus transfer are computed using finite element analysis. The transitions are studied at several geometries by varying the radii of neuron and electrode. The results indicate a sharp transition in both sealing resistance and stimulus

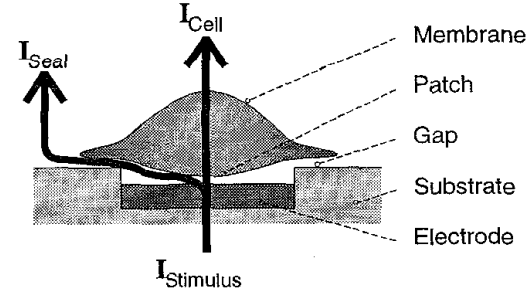

(a)

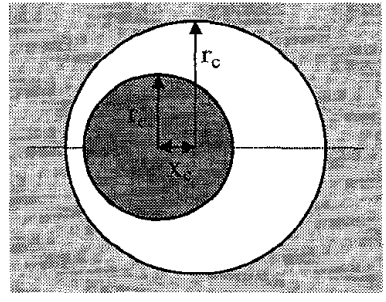

(b)

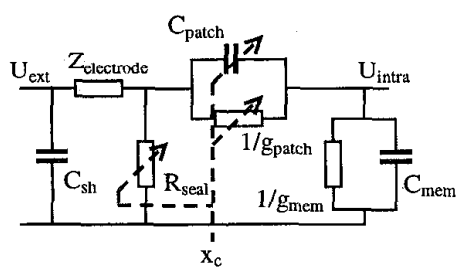

(c)

fig. 1 (a) Schematic presentation of the neuron-electrode interface. The efficiency of the stimulation current is increased by restriction of the leakage current through the gap. (b) Model assumption on the geometry of the neuron-electrode interface: the neuron is positioned excentrically on the electrode (with excentricity $x_{c}$ ). (c) Electrical equivalent circuit of the (passive) neuron-electrode interface. The sealing resistance and area of the patch membrane are modulated by the excentricity, $x_{\sigma}$, of the neuron. 
transfer around $x_{c}=r_{c}-r_{e}$. In case of complete sealing the stimulus transfer remains relatively constant.

\section{METHODS}

In fig. $2 \mathrm{a}$, the simplified geometry of the neuron-electrode interface is depicted. The neuron is modeled as a pillbox shaped cell of radius $r_{c}=7 \mu \mathrm{m}$, positioned with an excentricity $x_{c}$, on top of an electrode of radius $r_{e}=5 \mu \mathrm{m}$. The electrode surface is represented by the bottom of a $800 \mathrm{~nm}$ deep hole. Between the lower membrane surface of the cell and the substrate, the sealing gap is modeled (but not over the electrode area).

The geometry of the neuron-electrode interface is implemented in ANSYS Finite Element Software release 5.3 (SAS IP inc., Houston). The medium, surrounding the neuron-electrode interface is modeled as a pillbox shaped volume conductor of conductivity $\sigma_{\mathrm{m}}=1.65 \mathrm{~S} / \mathrm{m}$, radius

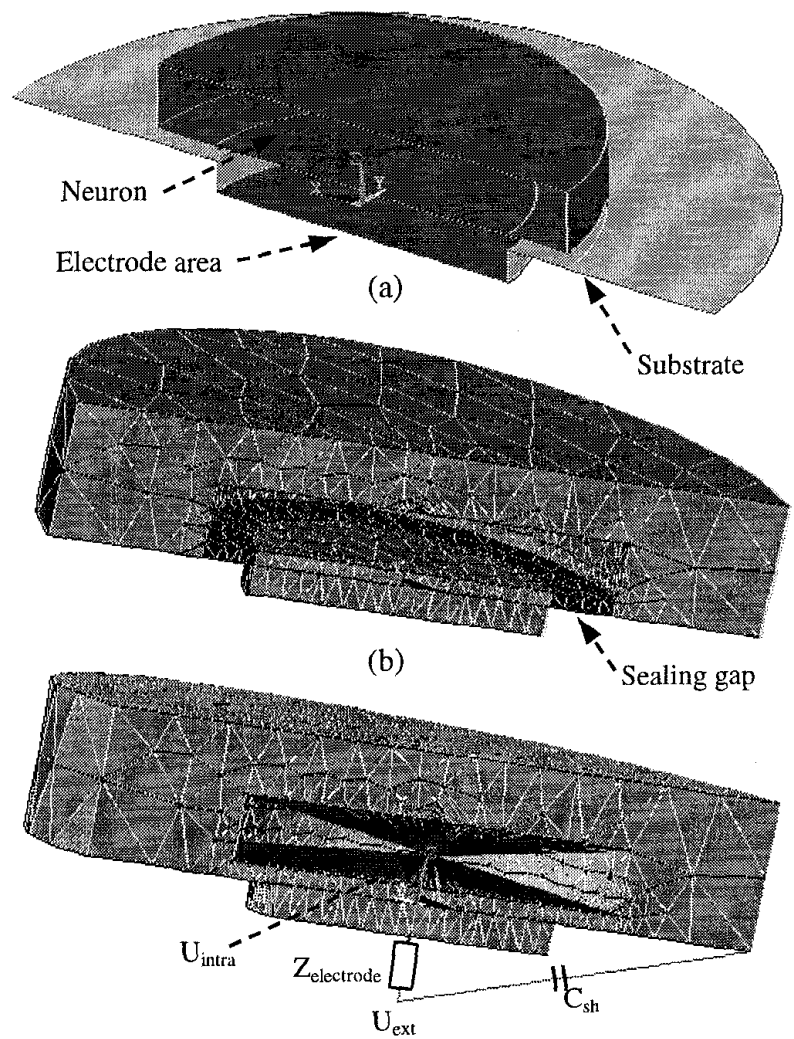

(c)

fig. 2 (a) Simplified geometry of the neuron-electrode interface. The neuron is modeled as a pillbox shaped cell and is positioned on top of an electrode with a small sealing gap between the cell membrane and the substrate. (b) Finite element model of the neuron-electrode interface.The neuron is modeled as a cavity inside a volume conductor respresenting the surrounding medium. The conductivity of the sealing gap is corrected for its actual thickness. (c) The intracellular space is assumed to be equipotential and therefore modeled with a single node.The membrane is represented by capacitive circuit elements, connecting the intracellular node to the nodes on the cell upper and lower surface. Finally, the electrode impedance and shunt capacity are added to the model using circuit elements. $r_{m}=r_{e}+r_{c}+\frac{1}{2} x_{c}$, positioned at $\frac{1}{2} x_{c}$. The neuron is modeled as a pillbox shaped cavity inside this volume conductor (fig. 2b). The sealing gap could not be meshed when implemented with its actual thickness of $10 \mathrm{~nm}$ [5]. Therefore the conductivity of the gap (the dark ring in fig. 2b) was corrected for the difference between modeled and actual thickness.

All volumes in the model are meshed and filled with tetrahedral shaped volume elements of type SOLID98.

The intracellular potential is assumed constant over the entire intracellular space. Therefore, the intracellular potential can be represented by a single node. The electrical properties of the (passive) neuronal membrane are implemented by capacitive circuit elements connecting the neuronal membrane to this intracellular node (fig. 2c). All capacities are scaled to a value of $1 \mu \mathrm{F} / \mathrm{cm}$ using the area represented by the membrane nodes.

The electrode surface is modeled as an equipotential area by coupling all nodal potentials to one principal node in the area. A current applied to this principal node will result in an equivalent uniform current density over the entire electrode surface. The electrode-electrolyte interface impedance is added to the model by connection of circuit elements between the principal node of the electrode area and a contact node, representing $U_{\text {ext }}$ (see fig. 1c and fig. 2c). Finally, the shunt capacity (between the electrode lead and the medium, across the insulation layer) is modeled by a capacitive circuit element.

The nodes of the upper surface of the volume conductor (medium) and of the circular outer surface are set to zero potential. A unity current is applied to the contact node. The finite element problem is solved at frequencies $\mathrm{f}=300 \mathrm{~Hz}$, $500 \mathrm{~Hz}, 700 \mathrm{~Hz}, 1 \mathrm{kHz}, 3 \mathrm{kHz}, 5 \mathrm{kHz}, 7 \mathrm{kHz}, 10 \mathrm{kHz}$ and 20 $\mathrm{kHz}$, for cell excentricities $x_{c}=0$ up to $x_{c}=4.0 \mu \mathrm{m}$, in steps of $0.2 \mu \mathrm{m}$. The excentricities $x_{c}=1.8 \mu \mathrm{m}$ and $x_{c}=2.0 \mu \mathrm{m}$ are left out since a proper meshing could not be produced for these geometries.

From every solution the computed potentials at the contact node and the intracellular node are saved. Since unity current is applied to the contact node, the potential of the contact node, $U_{\text {ext }}$, equals the total impedance of the neuron-electrode system and the potential of the intracellular node, $U_{\text {intra }}$, equals the stimulus current transfer function.

\section{RESULTS}

In fig. 3 the transition from complete $\left(x_{c}<r_{c}-r_{e}\right)$ to defect sealing $\left(x_{c}>r_{c}-r_{e}\right)$ is illustrated by the locations of the equipotential surfaces in the volume conductor (sealing gap and medium). For convenience, the circuit elements modeling the membrane, the electrode impedance and the shunt capacity are left out. When the cell is positioned right on top of the electrode, $x_{c}=0$, all equipotential surfaces are located in the sealing gap, which indicates that the potential drops mainly over the sealing gap (fig. $3 \mathrm{a}$ ). At $\mathrm{x}_{\mathrm{c}}=1.6 \mu \mathrm{m}$, the sealing is still complete and the potential drop is still located in the sealing gap (fig. 3b). When the sealing becomes defect, at $x_{c}=2.2$ $\mu \mathrm{m}$, the potential drop is extended to the rest of the volume conductor, indicating a much larger leakage current (fig. 3c). 
The impedance loci (the imaginary part vs. the real part for each computed frequency) of the total neuron-electrode system are depicted in fig. 4a. The loci are grouped in two clusters, which indicate a sharp transition from complete to defect sealing. In fig. $4 \mathrm{~b}$, some results from previous measurements on cultured electrode arrays are redrawn from [4] in order to allow comparison with fig. 4a. The measured loci are grouped in clusters, similar to the clusters in the computed results. This does not surprise, since only a few electrodes in the culture were completely covered by a cell.

The clustering is also present in the computed transfer of the applied stimulus current to the intracellular potential, $\mathrm{H}_{c}(\mathrm{f})$, as can be concluded from the Bode diagram in fig. 4c. For stimuli in the frequency range from $100 \mathrm{~Hz}$ to $3 \mathrm{kHz}$ the transfer function decreases with a factor ten when the sealing becomes defect.

In fig. 5a the sealing resistance is depicted as a function of cell excentricity, $x_{c}$. When the sealing is complete $\left(x_{c}<2 \mu \mathrm{m}\right)$ the sealing resistance decreases only slowly. At the transition from complete sealing to defect sealing however, a steep descent occurs to a value of less than $100 \mathrm{k} \Omega$.

The relation between the stimulus transfer and the cell excentricity is depicted in fig $5 \mathrm{~b}$ for frequencies of $1 \mathrm{kHz}$ and $10 \mathrm{kHz}$ (these points are also marked in the Bode diagrams of fig. $4 \mathrm{c}$ ). At $\mathrm{f}=1 \mathrm{kHz}$, this relation is very similar to the relation between sealing resistance and cell excentricity. At $\mathrm{f}=$ $10 \mathrm{kHz}$, the stimulus transfer for complete sealing is lower than at $\mathrm{f}=1 \mathrm{kHz}$, but remains more constant until the sealing becomes defect.

From the information presented in fig. 5a and fig. 5b, the stimulus transfer can be related to the sealing resistance. These results are depicted in fig. 6 . The relation appears to be linear at $\mathrm{f}=1 \mathrm{kHz}$. However, at $\mathrm{f}=10 \mathrm{kHz}$ saturation occurs when the sealing resistance becomes higher than $2 \mathrm{M} \Omega$.

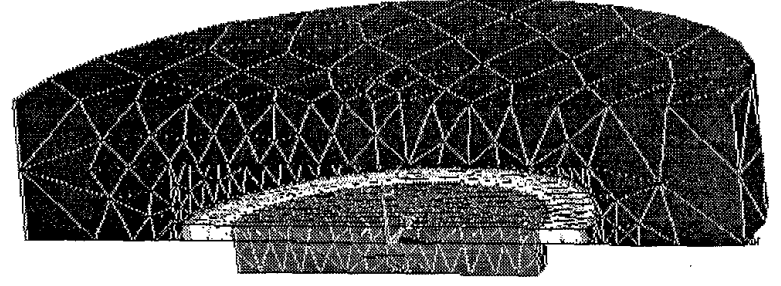

(a)

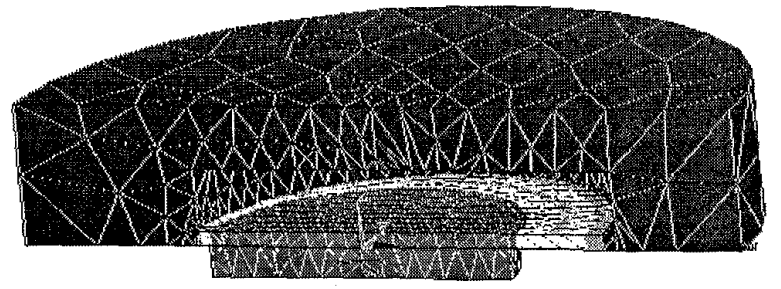

(b)

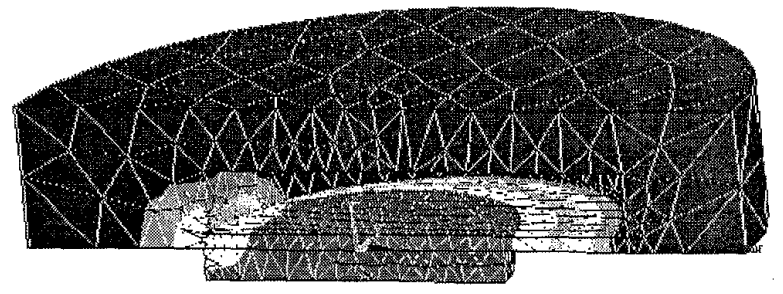

(c)

fig. 3 Illustration of the transition from complete to defect sealing. The colors mark the equipotential surfaces in the volume conductor. (a) $x_{c}=0$. Complete sealing $(b) x_{c}=1.8 \mu \mathrm{m}$. Still complete sealing $(c) x_{c}=2.2 \mu \mathrm{m}$. Defect sealing.

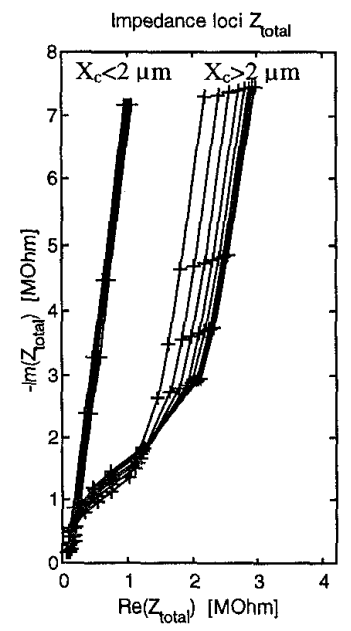

(a)

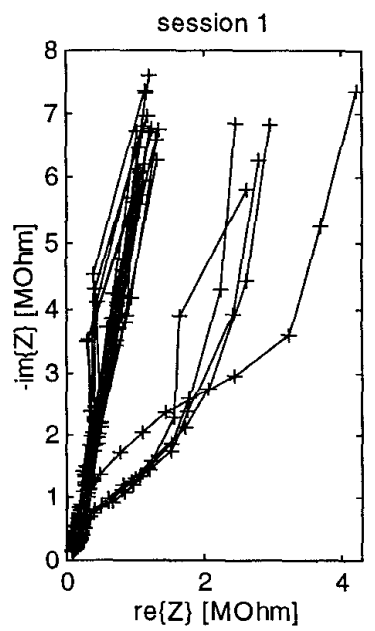

(b)

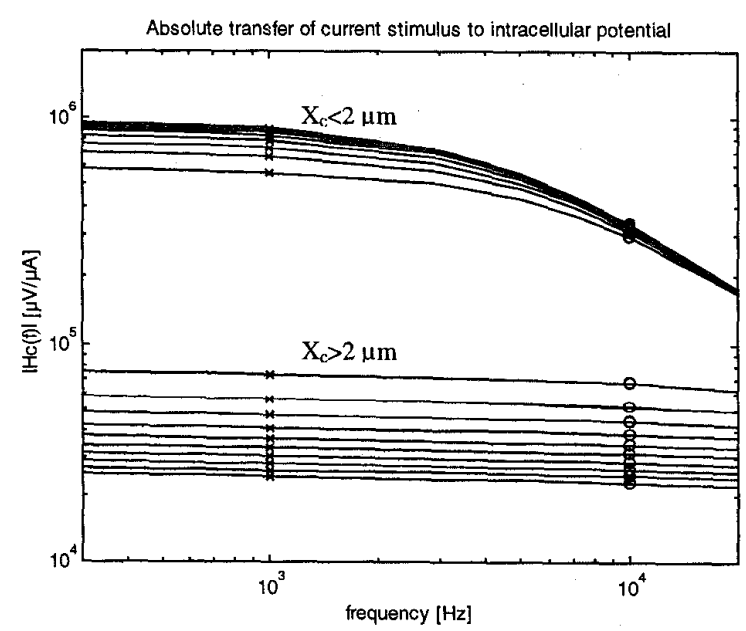

(c)

fig. 4 (a) Computed total impedance loci of the neuron-electrode system for several cell excentricities. The loci are grouped in two clusters: one for complete sealing and one for defect sealing. (b) Previously measured impedance loci from electrodes with cultured neurons (redrawn from [1]). The similarity in clustering with the computed loci is easily recognized. (c) Computed transfer from the applied stimulus current to the intracellular potential. The clusters of complete sealing and defect sealing are present. 


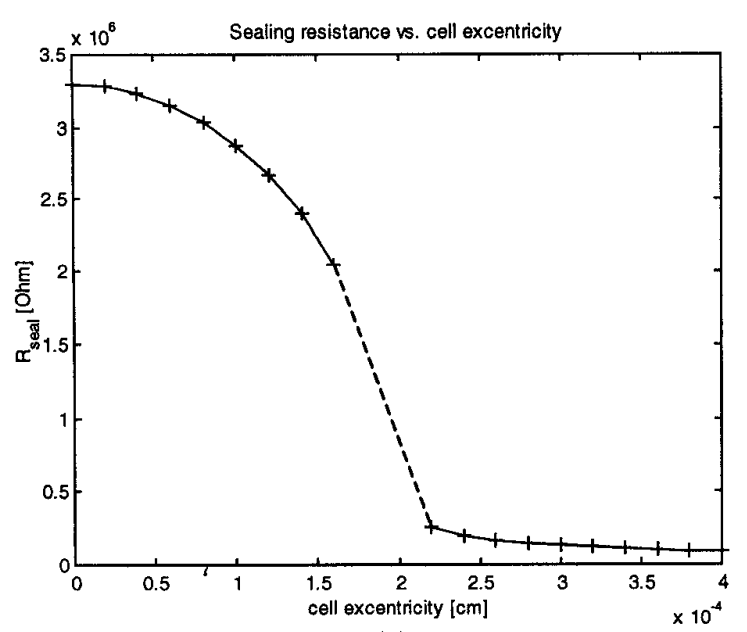

(a)

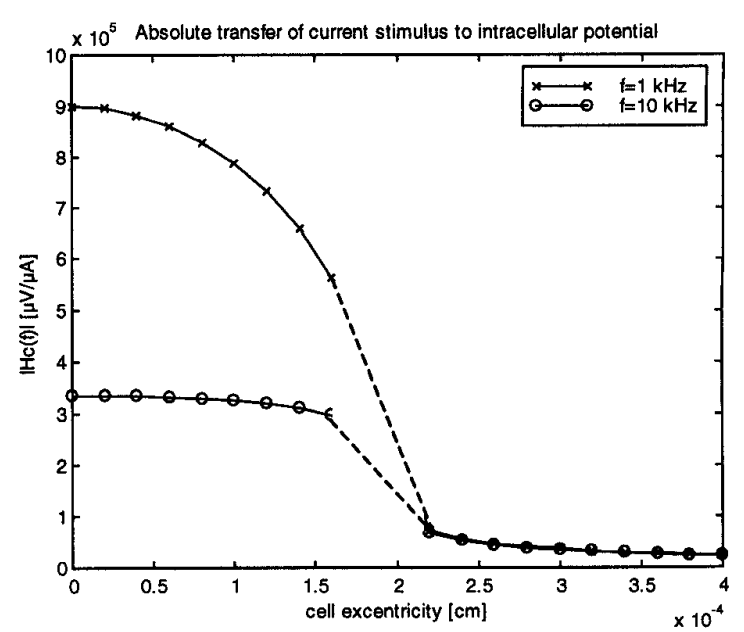

(b)

fig. 5 (a) Relation between sealing resistance and cell excentricity.During complete sealing, the sealing resistance remains relatively high and decreases only slowly with the excentricity. At the transition to defect sealing, the sealing resistance decreases to less than 100 $k \Omega$. (b)Relation between the stimulus transfer and the cell excentricity at two frequencies. At $f=1 \mathrm{kHz}$, the relation is very similar to the relation between sealing resistance and excentricity. At $f=10 \mathrm{kHz}$ the stimulus transfer is lower, but remains relatively constant until the sealing becomes defect.

\section{Discussion}

Although large simplifications are made in the representation of the geometry of the neuron-electrode interface, the impedance spectra, computed with the finite element model, show the same clustering as the measured impedance spectra. This clustering is also present in the computed stimulus transfer, which indicates that the finite element model provides a geometry induced relation between the sealing resistance and the stimulus transfer: even a small sealing defect annihilates both the sealing resistance and the stimulus transfer.

The highest stimulus transfer that can be reached is likely to depend also on the geometry of the neuron, to which large simplifications are made. This geometry will modulate the relation between sealing resistance and stimulus transfer. However, for an accurate geometry based prediction of the stimulus transfer, the finite element model can be refined

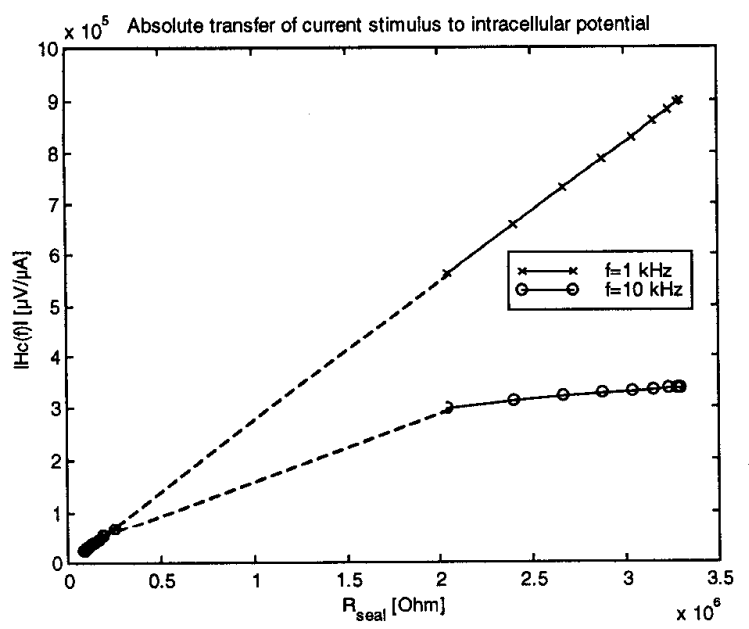

fig. 6 Relation between sealing resistance and stimulus transfer. At $f=1 \mathrm{kHz}$ this relation looks linear. At $f=10 \mathrm{kHz}$ the transfer saturates for sealing resistances higher than $2 M \Omega$ easily towards a more realistic representation of neuronal geometries.

\section{CONClusions}

The finite element approach offers a powerful method for linking the geometry of the neuron-electrode interface to its electrical properties. Small sealing defects annihilate both the sealing resistance and the stimulus transfer.

\section{ACKNOWLEDGEMENTS}

This work is part of the NESTING project (Neuro-Electronic Systems towards Tissue Implantation by Neural Grafting) and is supported by the BIOMED II EC project, shared cost contract no. BMH4-2723

\section{REFERENCES}

[1] W.G. Regehr, J. Pine, C.S. Cohan, M.D. Mischke and D.W. Tank, "Sealing cultured invertebrate neurons to embedded dish electrodes facilitates long-term stimulation and recording", J. Neurosc. Meth., 30, 91-106, 1989

[2] L.J. Breckenridge, R.J.A. Wilson, P. Connolly, J.A.T.Dow, S.E. Blackshaw and C.D.W. Wilkinson, "Advantages of using microfabricated extracellular electrodes for in vitro neuronal recordings", J. Neurosc. Res. 42, $266-$ 276, 1995

[3] M. Bove, M. Grattarola, S. Martinoia, G. Verreschi, "Interfacing cultured neurons to planar substrate microelectrodes: characterization of the neuron-to-microelectrode junction", Bioel. Chem. \& Bioen. 38, 255, 265, 1995

[4] J.R. Buitenweg, W.L.C. Rutten, W.P.A. Willems, J.W. van Nieuwkasteele, "Measurement of sealing resistance of cell-electrode interfaces in neuronal cultures using impedance spectroscopy", Med. Biol.Eng. Comp., in press, pp. 9, 1998 [5] R. Ehret, W. Baumann, M. Brischwein, A. Schwinde, K. Stegbauer and B. Wolf, "Monitoring of cellular behaviour by impedance measurements on interdigitated electrode structures", Biosens.\&Bioelectr., 12(1), 29-41, 1997 